\title{
Toward the multiphoton parametric oscillators
}

\author{
G.Yu.Kryuchkyan and N.T.Muradyan \\ Institute for Physical Research, National Academy of Sciences, \\ Ashtarak-2, 378410, Armenia
}

\begin{abstract}
We propose novel types of parametric oscillators generating both threephoton and four-photon bright light which are accessible for an experiment. The devices are based on the cascaded down-conversion processes and consist of second-order media inserted in two-resonant mode cavity. Discussion of dissipation and quantum features of the system are performed by the quantumjump simulation method and concerns to the Wigner functions. The phasespace multistabilities and critical threshold behavior of three- and four-photon subharmonics are obtained.
\end{abstract}

PACS number(s): 42.50.Lc, 42.65.S, 42.50.Dv, 42.65.Yj

Typeset using REVTEX 


\section{THE PROBLEM}

Optical parametric oscillators (OPO's) based on processes of down-conversion in a cavity have proved to be efficient sources of light with a range of unique properties. Among them it should be noted the nonclassical nature of light, including quantum correlations, subPoissonian statistics and a larger amount of squeezing [1]. OPO provides a method for producing tunable radiation from a coherent fixed-frequency pump source in the intracavity down-conversion, where a pump-photon splits into a pair of subharmonic photons. So far OPO has only been realized experimentally for two-photon down-conversion in nonlinear media with second-order $\chi^{(2)}$ susceptibility. Experimental and theoretical results can be found in [2].

In this letter we propose a new conception for the realization of multiphoton parametric oscillators using the idea of composite multiphoton interaction. Two schemes of OPO based accordingly on a three-photon and four-photon intracavity down-conversion are presented.

One of the principal motivations for the investigation of multiphoton OPO concerns to a production of nonclassical states of light with the novel features. Other motivation is their possible exploitation for quantum information technologies, because sources offering a great variety of multiparticle entangled states, first of all meaning photons, are required for the implementation of many quantum communication processes such as direct transmission of the state, quantum teleportation, quantum cryptography, and entanglement swapping [3].

It is known that two-photon down-conversion so far is the most standard source of pair of polarization-entangled photons which is relevant to many problems of quantum information (see, previous experiments on photon splitting in [4, and for polarization-entangled photon pairs [5]). Although two-particle entanglement has long been demonstrated experimentally the realization of entanglement between three or more particles has been considered as a difficult problem. Among the possible sources of three-photon states (so-called Greenberger-Horne-Zeilinger states [6]) we mention the process of degenerate three-photon down-conversion $\omega \rightarrow \omega / 3+\omega / 3+\omega / 3$ in a $\chi^{(3)}$ medium. This process has been studied for 
running waves [7]. Note, that most recently the polarization entanglement for three spatially separated photons has been observed [8]. Three-photon down-conversion in an optical cavity has also been proposed and investigated in [9], but too weak interaction between photons in $\chi^{(3)}$ medium makes experimental realization of this scheme a difficult problem. As regards to a four-photon splitting $\omega \rightarrow \omega / 4+\omega / 4+\omega / 4+\omega / 4$ in a $\chi^{(4)}$ medium, as we know, this process was not considered yet.

This letter reports the analysis of two schemes of multiphoton OPO in a double resonantmode cavity involving two crystals of second-order susceptibilities. They are the following:

\section{A. Four-photon splitting in cascaded down-conversion}

The scheme proposed contains two sequential nonlinear processes of degenerate twophoton splitting in $\chi^{(2)}$ media taking place inside the same cavity. This scheme for the special configuration when the coupling in- and out-fields occurs at one of the ring cavity mirrors is shown in Fig.1. The pump frequency $\omega$ is converted to the subharmonic frequency $\omega_{2}=\omega / 2$ and then into the frequency $\omega_{1}=\omega / 4$ in the cascaded parametric processes $\omega \rightarrow \omega_{2}+\omega_{2}$ and $\omega_{2} \rightarrow \omega_{1}+\omega_{1}$. We assume that the each of these processes takes place effectively only in the definite nonlinear crystal. The cavity is also assumed transparent at the pump frequency. So, we neglect the pump depletion effects considering the amplitude of driving field as a classical constant. We model the combination of these processes by the interaction Hamiltonian:

$$
H_{1}=i \hbar \chi_{1}\left(E a_{2}^{\dagger 2}-E^{*} a_{2}^{2}\right)+i \hbar k_{1}\left(a_{1}^{\dagger 2} a_{2}-a_{1}^{2} a_{2}^{\dagger}\right)
$$

where $a_{1}$ and $a_{2}$ are the operators of the modes $\omega_{1}$ and $\omega_{2}, \chi_{1}$ and $k_{1}$ are the nonlinear coupling constants for the processes $\omega \rightarrow \omega_{2}+\omega_{2}$ and $\omega_{2} \rightarrow \omega_{1}+\omega_{1}$ respectively which are related to second-order nonlinear susceptibilities $\chi^{(2)}$. $E$ is proportional to the coherent driving field amplitude. 


\section{B. Three-photon splitting in cascaded down-conversion}

This system mainly is similar to the previous one. The only difference is that the pump frequency $\omega$ converts to two different frequencies $\omega_{1}=\omega / 3$ and $\omega_{2}=2 \omega / 3$ in the parametric interaction $\omega=\omega_{1}+\omega_{2}$. The subharmonic $\omega_{1}$ is also produced in the other process of down conversion $\omega_{2}=\omega_{1}+\omega_{1}$. On the whole two subharmonic modes $\omega_{1}=\omega / 3$ and $\omega_{2}=2 \omega / 3$ are created in the cavity due to the cascaded processes. In the undepleted pump field configuration this model is described by the following interaction Hamiltonian:

$$
H_{1}=i \hbar \chi_{2}\left(E a_{1}^{\dagger} a_{2}^{\dagger}-E^{*} a_{1} a_{2}\right)+i \hbar k_{2}\left(a_{1}^{\dagger 2} a_{2}-a_{1}^{2} a_{2}^{\dagger}\right)
$$

where $a_{1}$ and $a_{2}$ are the operators of $\omega_{1}$ and $\omega_{2}$ subharmonics, and $\chi_{2}$ and $k_{2}$ are the coupling constants of the processes $\omega \rightarrow \omega_{1}+\omega_{2}$ and $\omega_{2} \rightarrow \omega_{1}+\omega_{1}$, respectively.

We note, that optical schemes involving cascaded nonlinearities in a cavity are currently of considerable interest and hold promise for applications including, in particular, frequency tunable sources of light, large third-order nonlinear effects via cascaded second-order nonlinearities and squeezing phenomena. The references and experimental observation can be found in [10]. Possible operational regimes of a combined system, as a rule, essentially differ from those in pure processes in each of nonlinear media. Some results in this direction are devoted to the cascaded frequency doubler [1]. Concerning our schemes, it is interesting to note, that the stable stationary above-threshold regime of oscillation is realized even in undepleted pump regime. The other novelty here is comparatively low values of the threshold of generation due to the schemes proposed can be accessible for experiments. Contrary to polarization-entangled state generation in two- and three-photon devices [5,8] the basic multiphoton states of our systems are neither based on polarization nor on momentum, but on phase-space entanglement of modes. 


\section{FOUR-PHOTON OPO}

The system of interest is dissipative because the subharmonic modes suffer losses due to partially transmission of light through the mirrors of the cavity. So that the reduced density operator $\rho$ of both modes obeys a master equation

$$
\frac{\partial \rho}{\partial t}=\frac{1}{i \hbar}\left[H_{1}, \rho\right]+\sum_{i=1,2} \gamma_{i}\left(2 a_{i} \rho a_{i}^{\dagger}-a_{i}^{\dagger} a_{i} \rho-\rho a_{i}^{\dagger} a_{i}\right)
$$

where $\gamma_{1}$ and $\gamma_{2}$ are the cavity damping rates for the modes $\omega_{1}$ and $\omega_{2}$.

To proceed with further analysis we present now the semiclassical steady-state solutions and stability properties of the system proposed. In the classical limit the system may be described by the complex-field amplitudes $\alpha_{1}$ and $\alpha_{2}$ of the resonant modes $\omega_{1}$ and $\omega_{2}$ whose real and imaginary parts respectively represent the dimensionless position and momentum. They obey the equation of motion:

$$
\begin{aligned}
& \frac{\partial \alpha_{1}}{\partial t}=-\gamma_{1} \alpha_{1}+2 k_{1} \alpha_{2} \alpha_{1}^{*} \\
& \frac{\partial \alpha_{2}}{\partial t}=-\gamma_{2} \alpha_{2}+2 \chi_{1} \alpha_{2}^{*} E-k_{1} \alpha_{1}^{2}
\end{aligned}
$$

Solving these equations for the steady-state regime and carrying out the standard linearized stability analysis one can arrive at the following results.

The trivial zero-amplitude solution $\alpha_{1}=\alpha_{2}=0$ is stable in the region $E<E_{t h}$, $E_{t h}=\gamma_{2} / 2 \chi_{1}$, with $E_{t h}$ being the threshold value of the pump field amplitude, and it describes the below-threshold regime of oscillation of both subharmonics. The stable abovethreshold solutions, for $E>E_{t h}$, expressed in terms of photon numbers $n_{j}$ and phases $\varphi_{j}$ of subharmonic modes $\left(\alpha_{j}=\exp \left(i \varphi_{j}\right) \sqrt{n_{j}}, j=1,2\right)$ are calculated as:

$$
\begin{aligned}
& n_{1}=\gamma_{1} \gamma_{2}\left(E / E_{t h}-1\right) / 2 k_{1}^{2}, \quad n_{2}=\gamma_{1}^{2} / 4 k_{1}^{2} \\
& \varphi_{1}=\frac{\Phi}{4} \pm \frac{\pi}{2} m, \quad \varphi_{2}=\frac{\Phi}{2} \pm \pi n, \quad n, m=0,1,2 \ldots
\end{aligned}
$$

where $\Phi$ is the phase of the driving field.

So, this scheme of multiphoton OPO allows us to reach stable generation of subharmonics at frequencies $\omega_{1}=\omega / 4$ and $\omega_{2}=\omega / 2$ at $E>E_{t h}$ in undepleted pump-field regime. It 
should be pointed out that the occurrence of such a regime of oscillation becomes possible just due to the combination of two kinds of down-conversion processes in a cavity. One of the peculiarities of cascading dynamics is that the threshold pump power $P_{t h}=c \hbar \omega E_{t h}^{2} / 2 L$ ( $L$ is the optical way of pump field) is only related to the coupling constant $\chi_{1}$ of $\omega \rightarrow$ $\omega / 2+\omega / 2$ parametric process. This coupling constant is proportional to the second-order susceptibility that leads to the comparatively low value of the threshold. We illustrate this fact for the system depicted in Fig.1, choosing realistic losses and typical values of $\chi_{1}$ and $k_{1}$ (see detailed calculations for a similar cavity configuration in [11]). Assuming $\chi_{1}=$ $k_{1}=3.4 \times 10^{4} s^{-1}, \gamma_{1}=2.4 \times 10^{8} s^{-1}, \gamma_{2}=3.6 \times 10^{8} s^{-1}$, we find for the threshold pump power $P_{t h}=74 \mathrm{~mW}$. For these parameters the power of the cavity output field of $\omega / 4$ mode increases as $P_{1}^{\text {out }}=2 \hbar \frac{\omega}{4} \gamma_{1} n_{1}=3.4 \times 10^{-3}\left(E / E_{t h}-1\right) W$, while the output power of the $\omega / 2$ subharmonic remains constant $P_{2}^{\text {out }}=2 \hbar \frac{\omega}{2} \gamma_{2} n_{2}=3.4 \times 10^{-3} \mathrm{~W}$.

A very important characteristic of multiphoton OPO concerning phase information of the generated modes, is displayed in phase-space. As we see from (6), the stationary states of $\omega / 4$ subharmonic have four-fold symmetry. There are four stable states of $\omega_{1}$ mode with equal photon numbers, but with four different phases which are: $\frac{\Phi}{4}, \frac{\Phi}{4}+\frac{\pi}{2}, \frac{\Phi}{4}+\pi, \frac{\Phi}{4}+\frac{3 \pi}{2}$. As regards to $\omega / 2$ subharmonic mode, it displays two-fold symmetry in phase-space, i.e. there exist two states with equal intensities but with different phases $\frac{\Phi}{2}, \frac{\Phi}{2}+\pi$.

Let us now show the general character of this symmetry in the frame of a full quantum treatment of interaction. It is easy to verify that the Hamiltonian (11) as well as the density operator $\rho$ obeying Eq.(3) satisfy the commutation relations

$$
\left[H_{1}, U\right]=[\rho(t), U]=0
$$

with the operator

$$
U=\exp \left(i \frac{\pi}{2} a_{1}^{\dagger} a_{1}+i \pi a_{2}^{\dagger} a_{2}\right)
$$

For the reduced density operators of each of the modes $\rho_{1(2)}=\operatorname{Tr}_{2(1)}(\rho)$, (which are constructed by tracing over one of the modes), relations ([]) give 


$$
\left[\rho_{1}(t), U_{1}\left(\frac{\pi}{2}\right)\right]=\left[\rho_{2}(t), U_{2}(\pi)\right]=0
$$

where operators $U_{i}(\varphi)=\exp \left(i \varphi a_{i}^{\dagger} a_{i}\right), i=1,2$ perform rotation by the angle $\varphi$ around the origin in phase-space of complex stochastic variables $\alpha_{1}$ and $\alpha_{2}$, respectively.

One of the most important conclusions of such symmetries is related to the Wigner functions $W_{1}$ and $W_{2}$ of the subharmonic modes $\omega_{1}$ and $\omega_{2}$ which provide a large amount of information about the states of the mode and also provide a pictorial view. To see this we address to the formula for Wigner function expressed through the density operator. Using also formulas (8) we arrive to the symmetries of Wigner functions written through the stochastic mode amplitudes $\alpha_{1}$ and $\alpha_{2}$ corresponding the operators $a_{1}$ and $a_{2}$. In the polar coordinates $r, \theta$ of the complex phase-space plane $X=\frac{\alpha+\alpha^{*}}{2}=r \cos \theta, Y=\frac{\alpha-\alpha^{*}}{2 i}=r \sin \theta$ these symmetries take the forms:

$$
W_{1}(r, \theta+\pi / 2)=W_{1}(r, \theta), \quad W_{2}(r, \theta+\pi)=W_{2}(r, \theta) .
$$

We have obtained this property of Wigner functions using the symmetry of Hamiltonian (11) and dissipation terms in master equation (3), assuming that the initial state of the modes is a vacuum state. Below, we will present the other confirmation to phase-space symmetry in quantum trajectories.

\section{THREE-PHOTON OPO}

The dynamics of subharmonic modes $\omega_{1}=\omega / 3$ and $\omega_{2}=2 \omega / 3$ for this system is governed by master equation (3) involving Hamiltonian (2) instead of Hamiltonian (11). Accordingly, the classical equations of motion for the complex amplitudes are

$$
\begin{aligned}
& \frac{\partial \alpha_{1}}{\partial t}=-\gamma_{1} \alpha_{1}+\chi_{2} \alpha_{2}^{*} E+2 k_{2} \alpha_{2} \alpha_{1}^{*}, \\
& \frac{\partial \alpha_{2}}{\partial t}=-\gamma_{2} \alpha_{2}+\chi_{2} \alpha_{1}^{*} E-k_{2} \alpha_{1}^{2} .
\end{aligned}
$$

We list below the semiclassical results for stable values of photon numbers and phases of the modes. In the above-threshold generation regime, at $E>E_{t h}$, where 


$$
E_{t h}=\frac{2 \sqrt{2 \gamma_{1} \gamma_{2}}}{3 \chi_{2}}
$$

is the threshold value of $\mathrm{E}$, the results take the form:

$$
\begin{aligned}
& n_{1}=\frac{\gamma_{1} \gamma_{2}}{18 k_{2}^{2}}\left(\varepsilon+3 \sqrt{\varepsilon^{2}-1}\right)^{2}, \\
& n_{2}=\frac{\gamma_{1}^{2}}{36 k_{2}^{2}}\left(\frac{\varepsilon+3 \sqrt{\varepsilon^{2}-1}}{\varepsilon+\sqrt{\varepsilon^{2}-1}}\right)^{2}
\end{aligned}
$$

where $\varepsilon=E / E_{t h}$,

$$
\phi_{1}=\frac{\Phi}{3}+\frac{2 \pi}{3} n, \phi_{2}=\frac{2 \Phi}{3}-\frac{2 \pi}{3} n, \quad n=0,1,2 .
$$

In the region $\varepsilon<\frac{3}{2 \sqrt{2}}$ the stability condition is fulfilled only for a zero amplitude steadystate solution $\alpha_{1}=\alpha_{2}=0$, i.e. the subharmonic field excitation exists at the spontaneous noise level. The photon numbers are plotted against the pump amplitude in Fig.2. They show bistable hysteresis-cycle behavior in a small domain $1<\varepsilon<\frac{3}{2 \sqrt{2}}$. The dashed parts of the curves correspond to unstable solutions. As we see, with increasing $E / E_{t h}$ the photon number of the $\omega / 3$ subharmonic increases as $n_{1} \simeq \frac{8 \gamma_{1} \gamma_{2}}{9 k_{2}^{2}} \varepsilon^{2}$, while the photon number of the $2 \omega / 3$ subharmonic goes to the saturated value $n_{2}=\gamma_{1}^{2} / 9 k_{2}^{2}$.

The very important peculiarity of the system proposed is that the threshold value $E_{t h}$ depends on the coupling constant $\chi_{2}$ which is related to the second-order $\chi^{(2)}$ susceptibility in contrary to the scheme cited in [9], where the threshold of the driving field amplitude is determined by $\chi^{(3)}$ susceptibility. However, the photon numbers of the generated modes depend on both nonlinear coupling constants $\chi_{2}$ and $k_{2}$. For the realistic parameters: $k_{2}=2.4 \times 10^{3} s^{-1}, \chi_{2}=2.3 \times 10^{3} s^{-1}, \gamma_{1}=0.9 \times 10^{8} s^{-1}, \gamma_{2}=1.2 \times 10^{8} s^{-1}$ parametric oscillations occur above the threshold pump power $P_{t h}=3 \mathrm{~W}$ and the threshold values of cavity-output field powers are $P_{1}^{t h} \approx 4 m W, P_{2}^{t h} \approx 4 m W$. Eqs.(12), (13) imply that in the above-bistability regime there exist three states for each of the modes which have equal intensities $n_{1}$ and $n_{2}$ but different phases: $\frac{\Phi}{3}, \frac{\Phi}{3}+\frac{2 \pi}{3}, \frac{\Phi}{3}+\frac{4 \pi}{3}$ and $\frac{2 \Phi}{3}, \frac{2 \Phi}{3}-\frac{2 \pi}{3}, \frac{2 \Phi}{3}-\frac{4 \pi}{3}$, respectively. Surprisingly, both the modes $\omega_{1}=\omega / 3$ and $\omega_{2}=2 \omega / 3$ have the identical threefold symmetry which also follows from the specific three-photon form of Hamiltonian 
(2). In the full quantum treatment of cascaded three-photon down-conversion this symmetry, in particular, is displayed in the Wigner functions. Following the method presented above, we have obtained:

$$
W_{i}(r, \theta+2 \pi / 3)=W_{i}(r, \theta), \quad i=1,2 .
$$

\section{QUANTUM SIMULATION: MULTISTABILITIES AND THRESHOLD BEHAVIOR}

The second part of this paper is devoted to the study of quantum-statistical properties of cascaded multiphoton OPO in a dissipative cavity. Our aim is to analyze the phase-space properties of the subharmonic modes in a quantum regime in the presence of quantum noise on the basis of the Wigner functions.

Note that an exact analysis of quantum optical nonlinear systems including dissipation effects is a difficult problem which was solved for a few single models (see [12] and references therein). In this field of research, analytical expression for the Wigner function of twophoton OPO was derived in [13]. However the quantum-statistical properties of cascaded OPO's can not be analyzed using single analytical formulas. Our approach is based on the quantum-jump simulation method also known as the state-vector Monte-Carlo method [14]. This method considers not the density matrix but deals with the state vector $\left|\Psi^{\alpha}(t)\right\rangle$ which is a member of the ensemble of state vectors and index $\alpha$ indicates the realizations. The

procedure adopted in the quantum-jump simulation for the system Hamiltonians (11) and (2) consists in the following. The evolution of a member of the ensemble of pure states $\left|\Psi^{\alpha}(t)\right\rangle$ over a short time $\delta t$ is governed by the non-Hermitian effective Hamiltonian if there is no quantum jump

$$
H_{1(2) e f f}=H_{1(2)}-i \hbar\left(\gamma_{1} a_{1}^{\dagger} a_{1}+\gamma_{2} a_{2}^{\dagger} a_{2}\right) .
$$

Such evolution must be completed by the possibility of quantum jumps which change the state vectors of both modes. On the whole the state transforms in the following way 


$$
\left|\Psi^{\alpha}(t+\delta t)\right\rangle \rightarrow \begin{cases}\left(1-\frac{i}{\hbar} \delta t H_{1(2) e f f}\right)\left|\Psi^{\alpha}(t)\right\rangle /(1-\delta p)^{1 / 2}, & \text { with probability } 1-\delta p \\ \sqrt{2 \gamma_{i}} a_{i}\left|\Psi^{\alpha}(t)\right\rangle /\left(\delta p_{i} / \delta t\right)^{1 / 2}, & \text { with probability } \delta p_{i}, i=1,2\end{cases}
$$

where $\delta p=\delta p_{1}+\delta p_{2}$.

We generalize the method presented in 15 for the quantum-jump simulation of the Wigner functions. Details of analogous calculations for the second harmonic generation can be found in [16]. The numerical simulations are performed in the truncated Fock basis of both subharmonic modes $\omega_{1}$ and $\omega_{2}$ in the regime of strong nonlinear coupling between the subharmonic modes, i.e. $\chi_{1,2} \lesssim \gamma_{1}, \gamma_{2}$ and $k_{1,2} \lesssim \gamma_{1}, \gamma_{2}$. We note that the OPO with such extremely large nonlinearities are not realized in practice and in this part of the Letter we do not intend to give results close to an experimental situation but discuss the fundamental problems of multiphoton entangled states.

To be short, we shall give the results for three-photon OPO, where one kind of phasespace symmetries is realized. The steady-state Wigner functions for $\omega_{1}=\omega / 3$ and $\omega_{2}=2 \omega / 3$ modes are calculated in the basis of $n=45$ photon number states for each mode. The results may be written as depending on the dimensionless parameters: $\varepsilon=E / E_{t h}, k_{2} / \gamma_{1}, \gamma_{2} / \gamma_{1}$. We calculate the Wigner functions of $\omega_{1}=\omega / 3$ subharmonic averaged over 1000 trajectories in the complex phase-space plane $X, Y$. It is obvious that below the threshold the Wigner function is single-humped and centered at $X=Y=0$. This state indicates appearance of three arms as the consequence of small entanglement between the modes. Increasing the driving field $E$ we enter the bistability domain and observe the occurrence of three additional side-humps. They correspond to the above-threshold steady-states (12), (13) with equal photon numbers and threefold symmetric phases. We see explicitly that in this domain the behavior of our system is actually four-stable. With further increase of $E / E_{t h}$ the central hump disappears, while the side-humps increase and we turn to a manifestly above threshold oscillation regime when we observe the phase space three-stability. This case is shown in Fig.3 for the parameters $k_{2} / \gamma_{1}=0.2, \gamma_{2} / \gamma_{1}=0.4, \varepsilon=1.59$. Analogous 
behavior has the Wigner function of $2 \omega / 3$-subharmonic.. The difference between the results of two modes is displayed in the form of squeezing the humps.

\section{CONCLUSION}

Thus we have shown that the idea of using the cascaded photon splitting processes in a cavity opens a novel route for the realization of a multiphoton parametric oscillator. Two devices are proposed which transform the pump coherent field into two subharmonics at

frequencies $\omega / 2$ and $\omega / 4$ (configuration A) and $\omega / 3$ and $2 \omega / 3$ (configuration B). Furthermore, these models of $\mathrm{OPO}$ are to be subject to an experiment. We have been convinced that the pump threshold of each of the OPO is only expressed through the second-order susceptibility. As a result, comparatively low values of thresholds have been obtained for typical parameters. The important features of multiphoton OPO obtained are phase-space symmetries and multistabilities of subharmonics. In addition, it is shown that subharmonics "remember" the phase of the pump field. Our numerical quantum Monte-Carlo simulations display phase-space multistabilities and threshold behavior of subharmonics in the presence of dissipation.

\section{Acknowledgments}

We acknowledge the helpful discussions with J.Bergou, H.Carmichael, S.Fauve and L.Manukyan. The work was supported in part by INTAS grant No 97-1672, and by grant No 00375 awarded by the Armenian Science Foundation. 


\section{REFERENCES}

[1] See, Special issue on squeezed states of light, J. Opt. Soc. Am. B 4, (1987); M.C.Teich, B.E.Salen, Prog. in Opt. 26, 3 (1988)

[2] P.D.Drummond, K.J.McNeil, and D.F.Walls, Opt. Acta 27, 321 (1980); 28, 211 (1981); L.-A.Wu, K.J.Kimble, J.L.Hall, H.Wu, Phys. Rev. Lett. 57, 691 (1986); A.Heidmann, R.J.Horowicz, S.Reynaud, E.Giacobino, C.Fabre, Phys. Rev. Lett. 59, 2555 (1987); A.S.Lane, M.D.Reid, and D.F.Walls, Phys. Rev. A 38, 788 (1988); G.Breitenbach, T.Muller, S.F.Pereira, J-Ph.Poizat, S.Schiller, and J.Mlynek, J. Opt. Soc. Am. B 12, $2304(1995)$

[3] S.J.Euk, J.I.Cirac, and P.Zoller, Science 279, 205 (1998); C.H.Bennett, et al., Phys. Rev. Lett. 70, 1895 (1996); D.Bouwmeester, J.-W.Pan, K.Mattle, M.Eible, H.Weinfurter, A.Zeilinger, Nature 390, 575 (1997); A.Furusawa et al., Science 282, 706 (1998); W.Tittel et al., Phys. Rev. A 57, 3229 (1998); J.-W.Pan, D.Bouwmeester, H.Weinfurter, A.Zeilinger, Phys. Rev. Lett. 80, 3891 (1998)

[4] D.C.Burnham, D.L.Weinberg, Phys. Rev. Lett. 25, 84 (1970); D.N.Klyshko, "Photons and Nonlinear optics", Nauka, Moskow (1980); S.Friberg, C.Hong, and L.Mandel, Phys. Rev. Lett. 54, 2011 (1985); Z.Y.Ou and L.Mandel, Phys. Rev. Lett. 61, 50 (1988); P.G.Kwait, A.M.Steinberg, and R.Y.Chiao, Phys. Rev. A 47, R2472 (1993); J.Brendel, E.Mohler, and W.Martienssen, Europhys. Lett. 20, 575 (1992)

[5] P.G.Kwait, K.Mattle, H.Weinfurter, A.Zeilinger, A.V.Sergienko and Y.Shin, Phys. Rev. Lett. 75, 4337 (1995); P.G.Kwait and A.G.White, proceeding of IQEC'98 (International Quantum Electronics Conference), eds. OSA, 144 (1998); D.Bouwmester et al., Nature (London) 390, 575 (1997); D.Boschi et al., Phys. Rev. Lett. 80, 1121 (1998)

[6] D.M.Greenberger, M.A.Horne, A.Zeilinger, Quantum Theory and Conceptions of the Universe, edited by M.Kafatos, (Kluwer Academics, Dordrecht, The Netherlands, 1989) pp.73-76; D.M.Greenberger et al., Am. J. Phys. 58, 1131 (1990); D.M.Greenberger, 
M.A.Horne, A.Zeilinger, Physics Today, 22, August 1993; N.D.Mermin, Physics Today, 9, June 1990

[7] S.L.Braunstein and R.L.McLachan, Phys. Rev. A 35, 1659 (1987); M.Hillery, Phys. Rev. A 42, 498 (1990); J.Bajer, J. Modern Opt. 38, 1085 (1991); S.L.Braunstein and C.H.Caves, Phys. Rev. A 42, 4115 (1990); G.Drobny and I.Jex, Phys. Rev. A 45, 4897 (1992); R.Tanas and Ts.Gantsog, Phys. Rev. A 45, 5031 (1992); I.Jex, G.Drobny, and M.Matsuoka, Opt. Comm. 97, 619 (1992); V.Buzek and G.Drobny, Phys. Rev. A 47, 1237 (1993); G.Drobny, I.Jex, and V.Buzek, Acta Phys. Slov. 44, 155 (1994); G.Drobny and V.Buzek, Phys. Rev. A 50, 3492 (1994); K.Banaszek and P.L.Knight, Phys. Rev. A 55, $2368(1997)$

[8] D.Bouwmeester and J.-W. Pan, Phys. Rev. Lett. 80, 3891-4 (1998); D.Bouwmeester et al., quant-ph/9810035, 1998

[9] T.Felbinger, S.Schiller, and J.Mlynek, Phys. Rev. Lett. 80, 492 (1998)

[10] A.G.White, P.K.Lam, M.S.Taubman, M.A.M.Marte, S.Schiller, D.E.McClelland, and H.-A.Bachor, Phys. Rev. A 55, 4511 (1997); G.Yu.Kryuchkyan, S.H.Karayan and N.T.Mouradyan, Opt. Comm. 159, 309 (1998)

[11] K.V.Kheruntsyan, G.Yu.Kryuchkyan, N.T.Muradyan, and K.G.Petrosyan, Phys. Rev. A 57, 535 (1998); G.Yu.Kryuchkyan, N.T.Muradyan and A.S.Sargsian, Opt. Comm. 146, $208(1998)$

[12] Yu.Kryuchkyan and K.V.Kheruntsyan, Opt. Comm. 127, 230 (1996)

[13] K.V.Kheruntsyan, D.S.Krahmer, Yu.Kryuchkyan, and K.G.Petrossian, Opt. Comm. 139, $157(1997)$

[14] J.Dalibard, Y.Castin, and K.Molmer, Phys. Rev. Lett. 68, 580 (1992); K.Molmer, Y.Castin, and J.Dalibard, J. Opt. Soc. Am. B 10, 524 (1993); H.Y.Carmichael, An Open Systems Approach to Quantum Optics, Lecture Notes in Physics (Springer-Verlag, 
Berlin, 1993)

[15] B.M.Garraway and P.L.Knight, Phys. Rev. A 49, 1266 (1994)

[16] S.T.Gevorkyan, G.Yu.Kryuchkyan, and N.T.Muradyan, Phys. Rev. A 61, 043805 (2000)

\section{Figure Captions}

Fig.1. Principal scheme of the cascaded parametric oscillator. The cavity is resonant at two frequencies $\omega_{1}=\omega / 4$ and $\omega_{2}=\omega / 2$ of the subharmonics. The phase matching condition for the process $\omega \rightarrow \omega / 2+\omega / 2$ is satisfied in the first medium, and for the process $\omega / 2 \rightarrow \omega / 4+\omega / 4$ in the second medium.

Fig.2. The normalized mean photon numbers $n_{1} / n_{1}^{\text {th }}$ (curve1) and $n_{2} / n_{2}^{\text {th }}$ (curve 2 ) of $\omega / 3$ - and $2 \omega / 3$ - modes versus the scaled pump intensity parameter $\varepsilon^{2}=E^{2} / E_{t h}^{2}$. The threshold values of the photon numbers are $n_{1}^{t h}=\frac{\gamma_{1} \gamma_{2}}{18 k_{2}^{2}}$ and $n_{1}^{t h}=\frac{\gamma_{1}^{2}}{16 k_{2}^{2}}$. The dashed parts of the curves 1,2 , and zero-amplitude (curve 3 ) solutions for both modes describe the unstable steady-state solutions.

Fig.3. The steady-state Wigner function of $\omega / 3$ mode which demonstrate the phase-space multistability in the above-threshold range. 